\title{
Patterned carbon nanotubes as a new three-dimensional scaffold for mesenchymal stem cells
}

\author{
Verda Ceylan Bitirim ${ }^{\text {a, } 1}$, Gokce Kucukayan-Dogu ${ }^{\text {b, } 1}$, Erman Bengu ${ }^{\text {c }}$, Kamil Can Akcali a,d,* \\ a Department of Molecular Biology and Genetics, Bilkent University, 06800, Ankara, Turkey \\ ${ }^{\mathrm{b}}$ Institute of Engineering and Science, Material Science and Nanotechnology Graduate Program, Bilkent University, 06800, Ankara, Turkey \\ c Department of Chemistry, Bilkent University, 06800, Ankara, Turkey \\ d Bilgen, Bilkent University Genetics and Biotechnology Research Center, 06800, Ankara, Turkey
}

\section{A R T I C L E I N F O}

\section{Article history:}

Received 22 August 2012

Received in revised form 7 January 2013

Accepted 24 March 2013

Available online 1 April 2013

\section{Keywords:}

Vertically aligned carbon nanotube

Stem cell

Extra cellular matrix

Viability

Patterning

Collagen

\begin{abstract}
A B S T R A C T
We investigated the cellular adhesive features of mesenchymal stem cells (MSC) on non-coated and collagen coated patterned and vertically aligned carbon nanotube (CNT) structures mimicking the natural extra cellular matrix (ECM). Patterning was achieved using the elasto-capillary induced by water treatment on the CNT arrays. After confirmation with specific markers both at transcript and protein levels, MSCs from different passages were seeded on either collagen coated or non-coated patterned CNTs. Adhesion and growth of MSCs on the patterned CNT arrays were examined using scanning electron microscopy image analysis and 3-(4,5-dimethyl-2-thiazolyl)2,5-diphenyl-tetrazolium bromide (MTT) assays. The highest MSC count was observed on the non-coated patterned CNTs at passage zero, while decreasing numbers of MSCs were found at the later passages. Similarly, MTT assay results also revealed a decrease in the viability of the MSCs for the later passages. Overall, the cell count and viability experiments indicated that MSCs were able to better attach to non-coated patterned CNTs compared to those coated with collagen. Therefore, the patterned CNT surfaces can be potentially used as a scaffold mimicking the ECM environment for MSC growth which presents an alternative approach to MSC-based transplantation therapy applications.
\end{abstract}

(c) 2013 Elsevier B.V. All rights reserved.

\section{Introduction}

Stem cell research has gained tremendous pace in the last four decades and a wealth of information on their physiology has been gathered since then. Mesenchymal stem cells (MSCs), which are also called as bone marrow stromal cells, are a subset of adult progenitor cells and have the ability to differentiate into adipocytes, chondrocytes, osteocytes, and cardiomyocytes [1]. They are characterized by their morphological features and the expression of a number of cell surface marker genes. In recent years, owing to their multiple-lineage potentials and immune-privileged properties, MSCs have become a feasible and potential source for the cell-based therapy and tissue engineering applications due to their proliferative and differentiation capabilities [2]. Moreover, these cells do not induce immune reaction in the host which allows their usage in allogeneic transplantation [2].

A key issue in MSC based tissue engineering is to control the growth and differentiation of cells. Extracellular matrix (ECM) plays a crucial role in proliferation and differentiation of MSCs. In recent years, by using different scaffold proteins the transplantation of differentiated

\footnotetext{
* Corresponding author at: Department of Molecular Biology and Genetics, Bilkent University, 06800, Ankara, Turkey. Tel.: +90 312 2902418; fax: +90 3122665097. E-mail address: akcali@fen.bilkent.edu.tr (K.C. Akcali).

${ }^{1}$ Equal contributions of authors.
}

MSCs into damaged tissue such as bone and chondrocytes was accomplished [3]. However, use of natural ECM extracted from animal tissues as scaffold is limited by the dimension, form of the original tissue and the potential pathogen risk [4]. Therefore, there has been a tremendous demand to develop better materials and scaffolds which can closely mimic the surrounding native tissue in the last decade [5]. Surface properties of scaffolds were given a high priority due to the potential of developing new environments capable of stimulating the adhesion and proliferation of cells. Recently, numerous studies reported on the effects of topographical patterns on cell viability [6,7]. In this regard, patterning of scaffold surfaces at a nanometer scale is considered as a promising tool $[8,9]$. It is reported that gene expressions of fibroblast cells were found to be enhanced on relatively rougher surfaces [10]. Nevertheless, conflicting reports in the literature for the relationship between cell adhesion and surface topography do exist such as those reported by Kunzler et al. [11] and Gentile and co-workers [12].

Carbon nanotubes (CNT) have been proposed for many potential application areas due to their chemical stability, good electrical conductivity and mechanical strength. In recent years, CNTs have been also studied in biotechnology as a support material for cell growth in tissue engineering and bio-sensors [13,14]. However, there are extensive studies on the biocompatibility and cytotoxicity of CNTs. While cytotoxicity of CNTs $[15,16]$ was not a direct consideration in this work, it is worth mentioning that evidence for cytotoxicity of CNTs has not been reported for cases 
where CNTs attached to a substrate or limited concentrations of CNTs were used $[17,18]$. There are also similar reports on the cytotoxicity of CNTs on stem cells. Zhu et al. demonstrated that powdered multiwalled carbon nanotubes (MWCNT) induced DNA damage in mouse embryonic stem cells [19]. On the other hand, Lobo et al. [20] and Giannona et al. [21] have shown the adhesion and growth of fibroblasts on CNT arrays without any toxic effects if they are firmly attached to a substrate. Furthermore, CNTs have also been reported as a suitable scaffold material for the growth of cells due to their superb electrical conductivity and chemical stability [22]. Finally, patterned surfaces made by CNTs were found to be guiding the growth of MSCs and neural cells $[23,24]$.

The fundamental criterion for growth and differentiation of stem cells on ECM surfaces is their adhesion. Cell adhesion sets off the cell growth, survival and migration. The common media used for cell growth consists of protein fibers such as collagen and elastin which are roughly 10 to $300 \mathrm{~nm}$ in diameter. In biotechnology, MWCNTs can be considered as an alternative for collagen fibers due to their similar sizes [13]. The present study focuses on the growth and viability of MSCs on patterned CNT arrays. The cell counts and viability experiments show that MSCs preferred non-collagen coated CNT surfaces which can have a significant impact in the design and preparation of advanced CNT-based scaffolds.

\section{Materials}

\subsection{Synthesis and patterning of CNT arrays}

The vertically aligned CNT arrays were grown by the alcohol catalyzed chemical vapor deposition (ACCVD) method on oxidized Si (100) surfaces. The catalyst layers were applied on the oxidized Si surface before the synthesis of CNTs. First, $10 \mathrm{~nm}$ layer of Al was evaporated on Si surface as the diffusion barrier, and then this was followed by the electron beam evaporation of $1 \mathrm{~nm}$ Co layer. Finally, Co catalyst layer was capped by another $0.5 \mathrm{~nm}$ thick Al layer. Si substrates with the aforementioned layers were introduced into the ACCVD furnace for the growth of CNT arrays through reduction and reaction steps. The reduction step was conducted at $625{ }^{\circ} \mathrm{C}$ for 15 min under flowing $\mathrm{H}_{2}$ and $\mathrm{Ar}$ gases (20 sccm and $100 \mathrm{sccm}$, respectively). Then, the growth of CNT array was achieved through the reaction step in where ethanol was used as a carbon source under flowing $\mathrm{H}_{2}$ and $\mathrm{Ar}$ gases $(20 \mathrm{sccm}$ and $100 \mathrm{sccm}$, respectively) for $30 \mathrm{~min}$. Patterning was induced to the vertically aligned CNT arrays by using a dropper filled with deionized water. Following this step, some of the patterned CNT arrays were treated with $1 \mu \mathrm{g} / \mu \mathrm{l}$ sterilized collagen solution for every $\mathrm{cm}^{2}$ (approximately 10:1 weight ratio of collagen to $\mathrm{CNT}$ ) resulting in two separate groups of patterned CNT arrays: one non-coated and the other collagen coated. It has been shown in an earlier study that, a collagen/MWCNT composite scaffold was used for bone cells, and researchers observed improved cell attachment [13].

\subsection{Isolation and culture of MSCS}

MSCs were obtained from male 9-week old, 280-300 g SpragueDawley rats. The animals were permitted unlimited access to food and water at all times and were housed under controlled environmental conditions $\left(22^{\circ} \mathrm{C}\right)$ with a $12 \mathrm{~h} \mathrm{light}$ and $12 \mathrm{~h}$ dark cycle in the animal holding facility of the Department of Molecular Biology and Genetics at the Bilkent University. This study protocol complied with Bilkent University's guidelines on humane care and use of laboratory animals. Bone marrow heterogeneous cell population was collected from the femur and tibia by flushing with a $5 \mathrm{ml}$ syringe containing $10 \%$ fetal bovine serum (Hyclone) in Dulbecco's modified Eagle medium (Invitrogen) after the rats were sacrificed by cervical dislocation. The cells were cultured in plastic culture dishes with Mesencult Media (StemCell Technology) with 20\% supplement (StemCell Technology) and $1 \%$ penicillin-streptomycin solution (Hyclone) in a $5 \% \mathrm{CO}_{2}$ incubator at $37^{\circ} \mathrm{C}$. Twenty-four hours after plating, media of the tissue culture plates were changed and the non-adherent cells were removed. Thereafter, their media were changed every 4 days, after washing with sterile $1 \times$ phosphate buffered saline (PBS) prior to the change. At the 14 th day, $3 \times 10^{5}$ cells were seeded on CNT surfaces and stated as passage zero ( $\mathrm{P0}$ ) group. When MSCs became confluent, they were passaged for the second and third time and then they were seeded on patterned CNT surfaces. In the rest of the manuscript, these were stated as the first passage (P1) and the second passage (P2) groups, respectively.

\section{Methods}

\subsection{Characterization of CNT arrays}

Synthesized CNT arrays were displayed by scanning electron microscope (SEM; Carl Zeiss Evo 40) using variable vacuum mode around 40 $\mathrm{kPa}$ (under water vapor) and transmission electron microscope (TEM; JEOL 2100 F) using holey-carbon film supported grids. Raman analysis was carried out using a Jobin Yvon microscope with an Ar ion excitation laser $(\lambda ; 532 \mathrm{~nm})$. A $50 \times$ microscope objective was used to focus the laser beam and to collect the scattered light. Atomic force microscope (AFM; Nanomagnetics) was utilized for the characterization of surfaces. The surface roughness of vertically aligned CNT arrays was calculated using $10 \mu \mathrm{m} \times 10 \mu \mathrm{m}$ AFM image taken in the tapping mode.

\subsection{Total RNA isolation and reverse transcription}

MSCs were trypsinized and the total cellular RNA was isolated from the precipitate using the RNeasy Mini kit (Qiagen) according to the manufacturer's protocol with additional DNase treatment. The cDNAs were synthesized from the total RNA samples with the DyNAmo cDNA synthesis kit (Finnzymes) according to the manufacturer's protocol.

\section{3. $R T-P C R$}

cDNA amplification for $C D 90, C D 71, C D 45, C D 34, C D 29$ and $\beta$-actin were performed using DyNAzyme II (Finnzymes). The primers and product sizes were listed in Table 1 . The initial denaturation step was at $95{ }^{\circ} \mathrm{C}$ for $5 \mathrm{~min}$, followed by 30 (for CD90 and CD34), 35 (for $C D$ 71), 26 (for $C D 29$ and $C D 45$ ) and 25 (for $\beta$-actin) cycles of denaturation for $30 \mathrm{~s}$ for all genes at $94{ }^{\circ} \mathrm{C}$, annealing for $30 \mathrm{~s}$ at $55{ }^{\circ} \mathrm{C}$ (for $C D 90$, CD34), $60 \mathrm{~s}$ at $66{ }^{\circ} \mathrm{C}$ (for $C D 71$ ), $45 \mathrm{~s}$ at $60{ }^{\circ} \mathrm{C}$ (for $\beta$-actin) and $30 \mathrm{~s}$ at $60{ }^{\circ} \mathrm{C}$ (for $C D 29$ and $C D 45$ ), followed by extension for $30 \mathrm{~s}$ (for $C D 90$, $C D 34, D C 29, C D 45$ ), $40 \mathrm{~s}$ (for $\beta$-actin) and $45 \mathrm{~s}$ (for $C D 71$ ) at $72{ }^{\circ} \mathrm{C} . \mathrm{A}$ final extension at $72{ }^{\circ} \mathrm{C}$ for $5 \mathrm{~min}$ was applied to all the reactions.

\subsection{Protein isolation and quantification}

MSCs were scraped from the cell culture plates in $1 \times$ PBS and the precipitate was treated for $30 \mathrm{~min}$ on ice with a lysis buffer containing $0.05 \mathrm{M}$ Tris- $\mathrm{HCl}, 1 \times$ protease inhibitor, $0.25 \mathrm{M}$ sodium chloride and

Table 1

Primer sequences used in PCR amplification to characterize MSCs.

\begin{tabular}{ll}
\hline Gene & Sequence \\
\hline CD90 & (F) $5^{\prime}$-CCAGTCATCAGCATCACTCT-3' \\
& (R) $5^{\prime}$-AGCTTGTCTCTGATCACATT-3' \\
CD34 & (F) 5'-TGTCTGCTCCTTGAATCT-3' \\
& (R) 5'-CCTGTGGGACTCCAACT-3' \\
CD71 & (F) 5'-ATGGTTCGTACAGCAGCAGA-3' \\
& (R) 5'-CGAGCAGAATACAGCCATTG-3' \\
CD29 & (F) 5'-ACTTCAGACTTCCGCATTGG-3' \\
& (R) 5'-GCTGCTGACCAACAAGTTCA-3' \\
CD45 & (F) 5'-ATGTTATTGGGAGGGTGCAA-3' \\
B-actin & (R) 5'-AAAATGTAACGCGCTTCAGG-3' \\
& (F) 5'-CTGGCCTCACTGTCCACCTT-3' \\
& (R) 5'-GGGCCGGACTCATCGTACT-3' \\
\hline
\end{tabular}


$1 \%(\mathrm{v} / \mathrm{v})$ IGEPAL. Then, the lysate was centrifuged at $13000 \mathrm{rpm}$ and $4{ }^{\circ} \mathrm{C}$ for $20 \mathrm{~min}$. Protein concentrations of supernatants were determined with Bradford protein assay as described [25].

\subsection{Western blotting}

The proteins were separated on $8 \%$ SDS-PAGE and transferred to a polyvinylidene fluoride membrane. The membrane was blocked with blocking solution for $1 \mathrm{~h}$ at room temperature and was incubated at $4{ }^{\circ} \mathrm{C}$ for $16 \mathrm{~h}$ in antibody solution at a concentration of $1: 250$ for $C D 29$, 1:500 for $C D 34$, and 1:1000 for $\beta$-actin. Then horseradish peroxidaselinked secondary antibodies were applied for $1 \mathrm{~h}$ in blocking solution. Finally, Super Signal West Femto Maximum Sensitivity Substrate (Thermo Scientific) was applied to the membrane for 5 min and placed in an $\mathrm{X}$-ray film cassette and developed. The anti-CD29 antibody was purchased from Chemicon, and the anti-CD34 and actin antibodies were purchased from Santa Cruz.
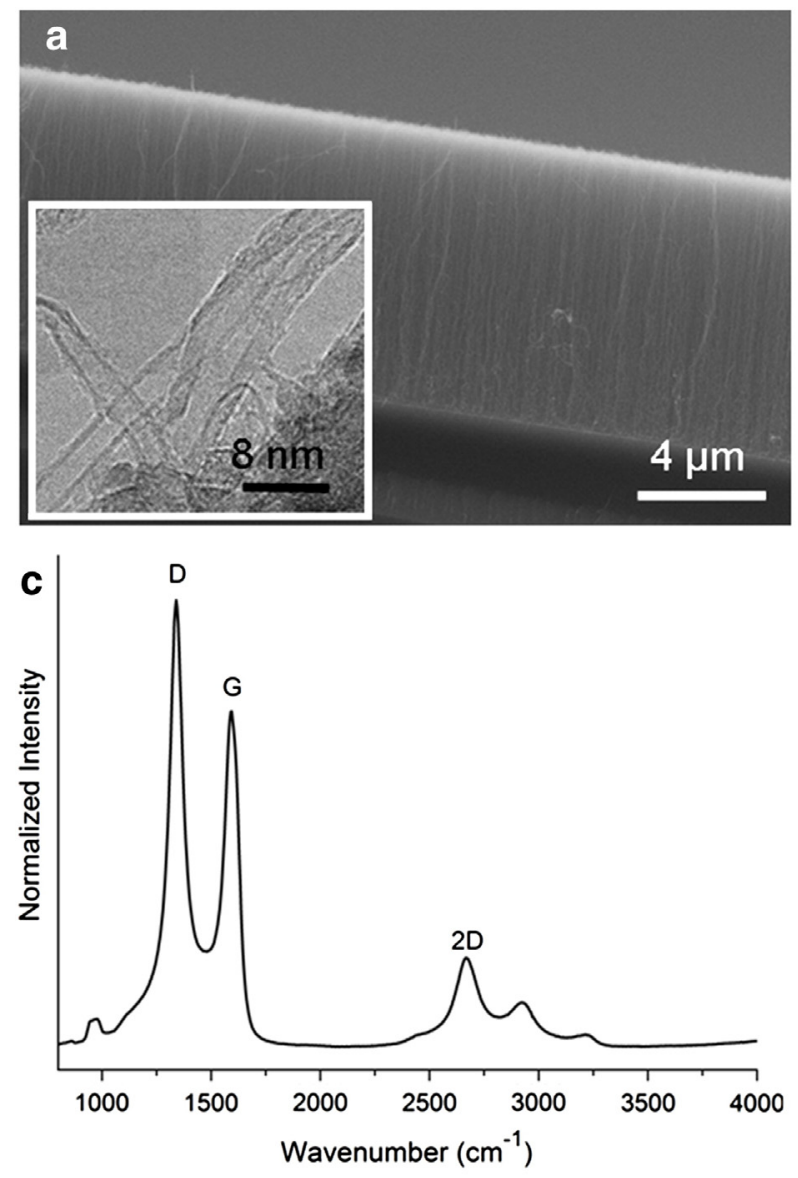

\subsection{Cell proliferation assay}

The viability of MSCs in the presence and absence of collagen was examined by cell proliferation assay (3-(4,5-dimethyl-2-thiazolyl)2,5-diphenyl-tetrazolium bromide- MTT) using Cell Proliferation Kit I (Roche). A total of $10^{4}$ numbers of MSCs were seeded onto patterned CNTs inside 96-well culture plates in the presence and absence of collagen and the assay was performed according to the manufacturer's protocol. Spectrophotometrical absorbance of the samples was measured by ELISA reader with the wavelength between 550 and $600 \mathrm{~nm}$.

\subsection{Statistical analysis}

All data were expressed as mean \pm standard deviation (SD) and the results were analyzed with ANOVA. Multiple comparisons of control to other groups were performed using Fisher's test (Minitab 15
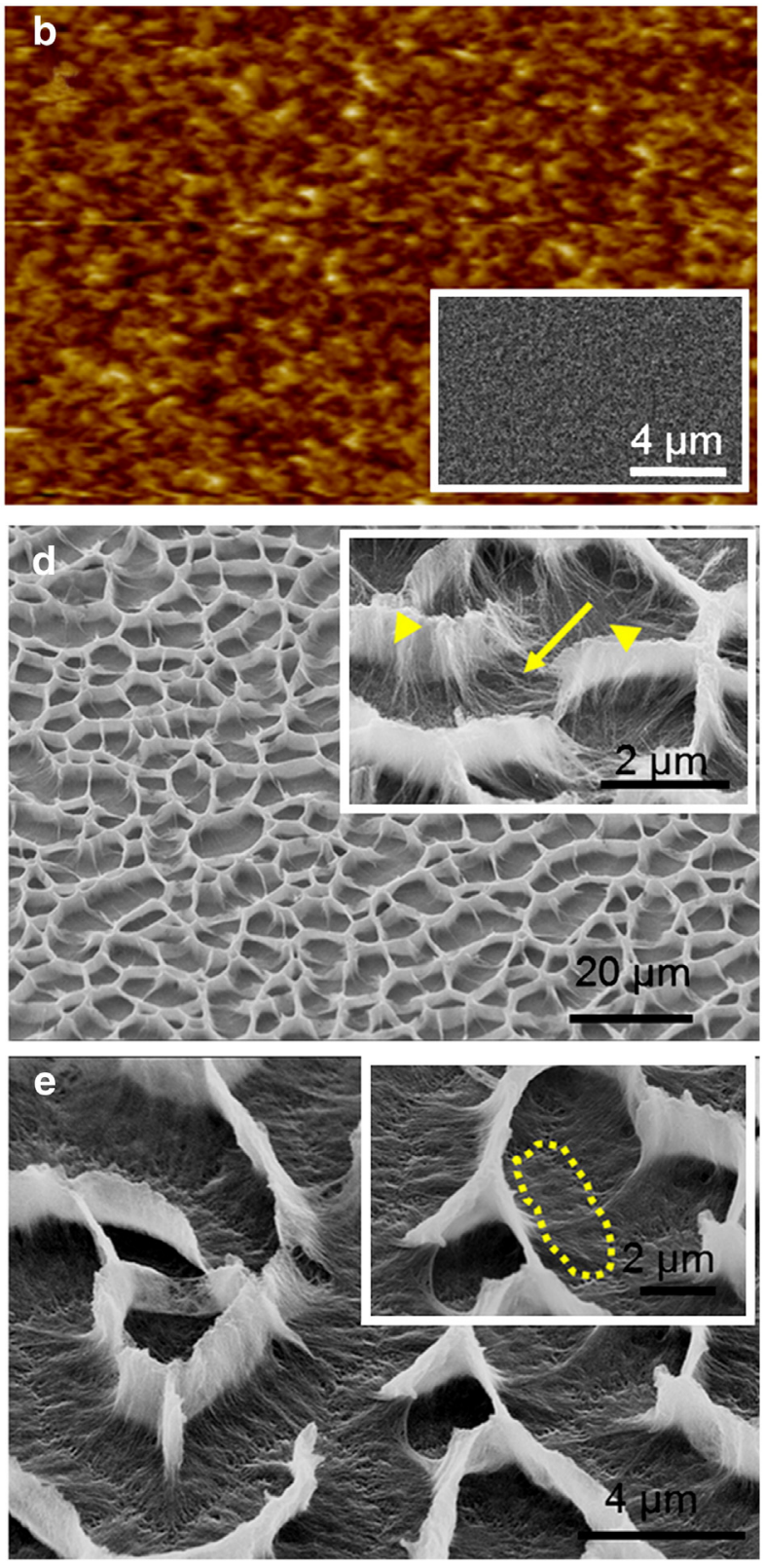

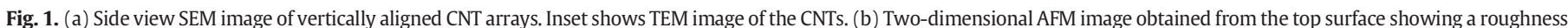

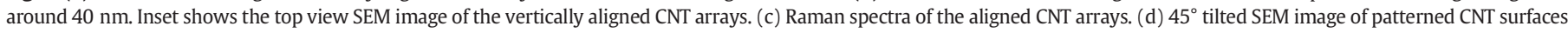

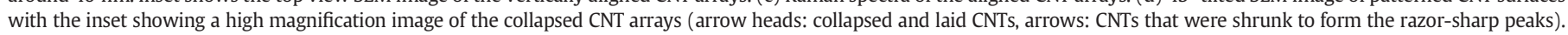
(e) $45^{\circ}$ tilted SEM image of the collagen coated patterned CNT surface with the inset showing the smoothing/leveling effect of collagen coating (dotted lines). 
Statistical Software). On all statistical tests, $p<0.05$ was regarded as significant difference.

\section{Results and discussion}

\subsection{Experimental design and characterization of CNT arrays}

We used SEM, TEM, Raman spectroscopy and AFM to characterize the vertically aligned CNT arrays (Fig. 1). Our SEM images of the vertically aligned CNT arrays on a $\mathrm{Si}(100)$ surface showed that CNTs were approximately $10 \mu \mathrm{m}$ in height (Fig. 1a). We also confirmed that CNTs were multi-walled with an average diameter of $10 \mathrm{~nm}$ by using TEM (Fig. 1a, inset). In order to investigate the top surface roughness of the vertically aligned CNT array, we used AFM (Fig. 1b). Two-dimensional $10 \mu \mathrm{m} \times 10 \mu \mathrm{m}$ AFM image showed that the root-mean-square roughness value for the surfaces was around $40 \mathrm{~nm}$. The smoothness of the CNT array top surface was also confirmed by using SEM (Fig. 1b, inset). Finally, by using Raman spectroscopy, the presence of CNTs was verified (Fig. 1c). The D (1344 $\left.\mathrm{cm}^{-1}\right)$ and $\mathrm{G}\left(1582 \mathrm{~cm}^{-1}\right)$ bands were corresponding to disorder and in plane vibration of carbon atoms, respectively. In addition, absence of radial breathing mode bands indicated that these were multi-walled variety.

Our experimental design was summarized in Fig. 2. First, the vertically aligned CNT arrays were synthesized by the ACCVD method (Fig. 2, step A). Then deionized water was applied on these surfaces with a dropper to create cavities in the patterning step (Fig. 2, step B). CNT arrays were either treated with or without collagen (Fig. 2, step $C^{\prime}$ and $C$; respectively) and MSCs from rat bone marrow were seeded (Fig. 2, step $\mathrm{D}$ and $\mathrm{D}^{\prime}$ ).

Presence of the asperities on the surface is critical for the preparation of a suitable scaffold for anchoring of the cells. Hence, we undertook the patterning step on the vertically aligned CNT arrays synthesized by ACCVD method in order to create a suitable so-called nest for the cells (Fig. 2, step A). To accomplish this task, we applied deionized water on the vertically aligned CNT arrays with a dropper and left for air drying (Fig. 2, step B). Due to capillary forces exerted by water, the vertically aligned CNT arrays patterned with the collapse of CNTs in equiaxed cavities/pockets, as shown in Fig. 1d. Similarly, self-assembled patterning of CNT arrays were also reported by another group [26]. The main mechanism of this formation is thought to be related to the hydrophobic properties and elasto-capillary effect of aligned CNTs. The size of the CNT cavities on the surface was around $10 \mu \mathrm{m}$ wide (Fig. 1d). Furthermore, patterned CNT arrays were either treated with or without collagen (step $\mathrm{C}^{\prime}$ and $\mathrm{C}$ in Fig. 2, respectively). Fig. 1d, 1e and related insets show the non-coated cavities and collagen coated cavities in a comparative manner. Finally, MSCs from rat bone marrow were seeded on the patterned non-coated and collagen coated CNT arrays (Fig. 2, step $\mathrm{D}$ and $\left.\mathrm{D}^{\prime}\right)$.

\subsection{Characterization of MSCS}

After the isolation from rat bone marrow, we characterized the MSCs both at mRNA and protein levels for P0, P1 and P2 groups (Fig. 3). Consistent with previous observations by us and others $[27,28]$, our results showed that MSCs were positive for the expression of MSC markers (CD90, CD71 and CD29) but negative for hematopoietic stem cell markers ( $C D 45$ and $C D 34)$ for all passages as expected at the transcript level (Fig. 3a). We further confirmed that these MSCs were positive for CD29 and negative for CD34 by Western blot analysis at the protein level (Fig. 3b).

\subsection{Seeding MSCs on the patterned CNT arrays}

A total of $3 \times 10^{5}$ numbers of MSCs were seeded on the noncoated and collagen coated patterned CNT surfaces. After 3 days, SEM analysis was performed (Fig. 4). Attached MSCs, which were from P0, were observed both in collagen coated (Fig. 4a) and non-coated (Fig. $4 \mathrm{~b}$ ) patterned CNT surfaces. In addition, numbers of attached MSCs on coated and non-coated patterned CNT surfaces were counted in the SEM images for three subsequent passages and average areal density was calculated dividing this number to the total surface area (Fig. 4c). Our plot revealed that the number of the MSCs decreased through the passage of the cells. The decrease was statistically significant when the number of the cells was compared between P0 and P1 and P2 (Fig. 4c). Thus, these data suggest that very early passages of MSCs should be used for seeding purposes. The number of the cells both on coated and non-coated patterned CNTs continued to decrease at P2 compared to P1 but we did not observe a statistical significance between them. Interestingly, the number of the MSCs was significantly higher when they were cultured on non-coated compared to that of collagen coated CNT arrays at P0 but not at P1 and P2 (Fig. 4).

Recent literature on collagen/CNT composites prepared and analyzed for use in scaffold applications reveals observations that beg further investigation. First of all, the goal for the development of such composite structures is not only to improve the poor mechanical properties of collagen, but also to add further functionality to the

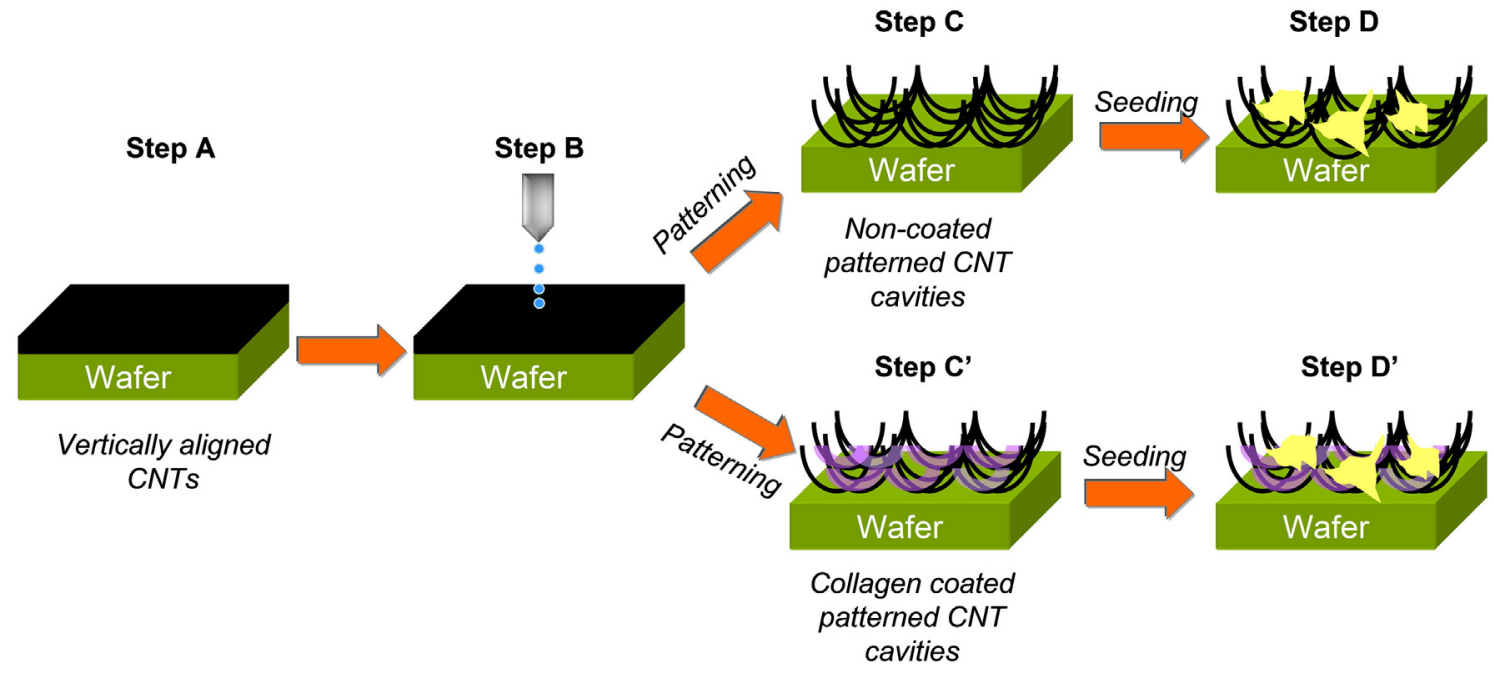

Fig. 2. Schematic representation of experimental approach. 
a

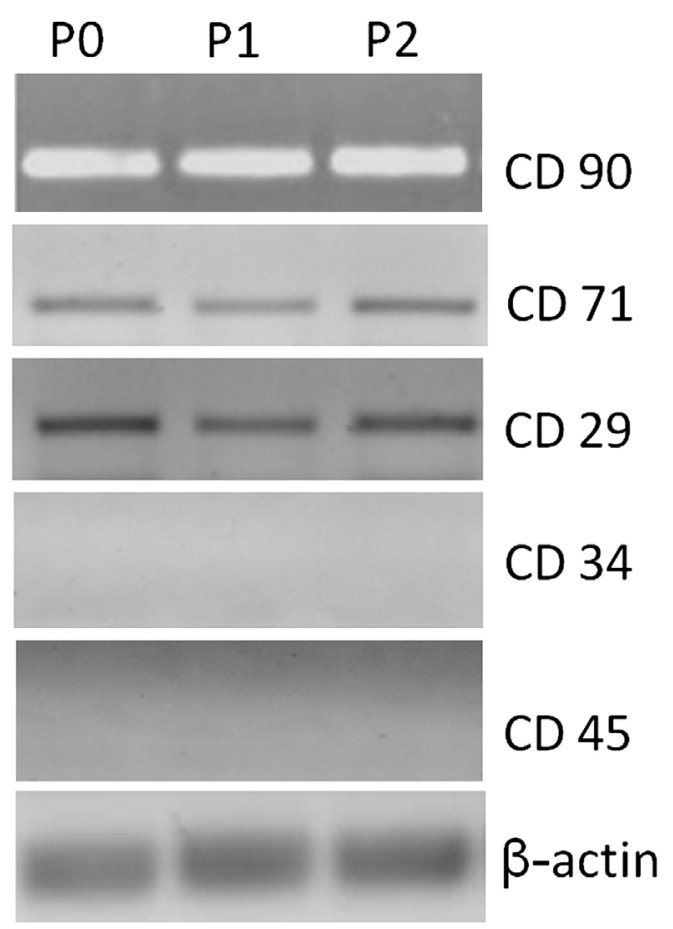

b

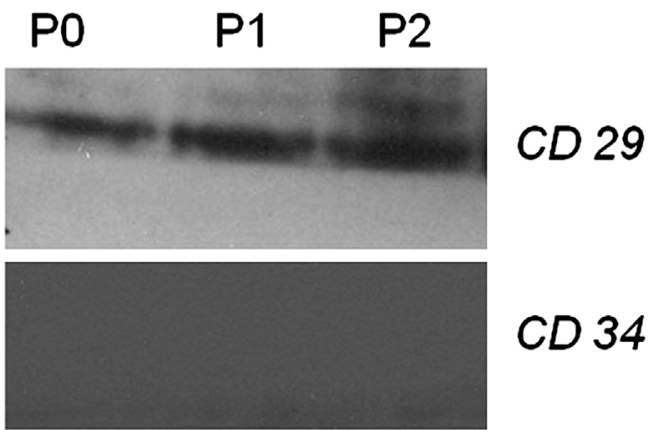

Fig. 3. The expression of the markers for bone marrow derived MSCs and hematopoietic stem cells at (a) mRNA and (b) protein levels.

scaffold $[9,22]$. For instance, Cho and Borgens prepared electrically conductive collagen/MWCNT composite structures and investigated the effect of CNT loading and applied electrical stimuli on the cell viability of PC12 [29]. Their findings indicated that the metabolic activity of these cells decreased asymptotically by the CNT amount reaching a plateau at around 50\% (wt.) CNT loading. However, there was no mention of a root cause for this observation. In contrast, our results shown in Figs. 4c and 5 clearly indicated that the number of MSCs for P0 was noticeably larger for the collagen coated patterned CNT surfaces compared to non-collagen coated ones. Concurrently, other studies also reported the improvement in the viability and the proliferation of different type of cells with the addition of CNT to the matrix which was ascribed to better attachment of the cells to the CNTs $[30,31]$. Hirata et al. showed that mouse osteoblast cells (MC3T3-E1) attached to a three-dimensional collagen sponge coated with MWCNTs exhibited higher DNA content than the uncoated collagen sponge [30]. In addition, MacDonald and co-workers showed an increase in the number of viable smooth muscle cells by increasing the mass ratio of CNT in collagen matrix [31]. One possible explanation for the MSCs preference of non-coated patterned CNT arrays could be related to the mechanical properties of ECM which was known to have a significant impact on the attachment of stem cells [32,33]. Collagen by itself has poor mechanical properties limiting its widespread usage in bioengineering [34]. On the other hand, MWCNT is one of the strongest materials known with a modulus of elasticity and tensile strength surpassing those of the strongest carbon fibers [35].

As mentioned before, cell attachment to the ECM is also affected by surface topography $[6,7,11]$. We speculated that the collagen infiltrated to the CNT array was acting as a smoothing agent filling the gaps between the CNTs, thereby limiting the availability of the anchoring points for attachment of MSCs. When collagen was dropped on the patterned CNT surface, the gaps between the collapsed CNTs were filled by collagen and we called this as 'smoothing'. The SEM images provided in Fig. 1d and e, display the comparison of the as-is and collagen infiltrated CNT array surfaces where the gaps between CNTs were coated by collagen. Hence, regarding these images, roughness for as-is surfaces was different than collagen coated ones. Another important aspect of the study presented in this work is the use of dense $\left(>10^{9} / \mathrm{cm}^{2}\right)$ vertically aligned CNT arrays. Such alignment of the CNTs not only provided a homogenous surface minimizing local variations in the mechanical properties, but at the same time presented numerous anchoring points to the cells. Besides, in this study the vertically aligned CNT arrays were firmly attached to the substrate beneath limiting the possible cytotoxic effects $[20,21]$.

Toxicity of CNTs has been a controversial issue. There are reports claiming toxic effect of CNTs $[17,18]$, whereas many reports with opposite views exist $[20,21]$. It has been shown that proliferation and differentiation of rat bone marrow derived MSCs were inhibited by MWCNTs [36]. Therefore, it was critical to test whether our modified and patterned CNT arrays would have any deleterious effect on the cell viability or not (Figs. 4 and 5). In addition we have examined the effect of MWCNTs on different passages. For this purpose, we performed MTT assay to measure their viability on both non-coated and collagen coated patterned CNT arrays in different passages (Fig. 5). Our results showed that MSCs from early passages (P0) had not only the highest capacity of viability both on non-coated and collagen coated patterned CNT surfaces, but also had capacity higher than the control groups. Also, the increase of cell number for PO on the non-coated patterned CNT surface (P0-CNT) compared to control was statistically significant. It is important to note that the reading of control group on MTT assay was obtained from cells on 96-well culture plates without CNT arrays. Previously, it was shown that less than $40 \%$ cell viability at the MTT results suggested that the tested material is toxic [37] and should be further investigated with other methods. Our data in Fig. 5 clearly showed that MSC viability is more than that of control at P0 and approximately 70\% to that of control group at P1 and 50\% to that of control group at P2. Therefore, we can state that there is no cytotoxic effect of both coated and non-coated CNTs on the MSCs at PO as well as in other passages. Thus, our data revealed that either non-coated or collagen coated patterned CNT arrays are providing a suitable environment for cells from early passages to survive and keep their differentiation potential. This should be remembered when scaffolds composed of CNTs are to be used for seeding cells. This may have important regenerative medicine applications. Our data also demonstrated the negative effect of CNTs on the number and metabolic activity of MSCs on later passages. MSCs in later passages (P1 and P2) indicating lesser counts (Fig. 4c) and viability (Fig. 5) on collagen coated or non-coated patterned CNTs than in early passage (P0) can be explained by the aging of MSCs $[27,38,39]$. Gruber et al. have shown that the mean percent senescence increased significantly with cell passaging [38] and high passage numbers adversely affected the functioning of stem cells [39].

While our results showed improved cell attachment and viability of MSCs on non-coated patterned CNT arrays, the need of a rigid surface such as Si for the growth of CNTs is a significant limiting factor for technological applications. The natural next step in such a study 

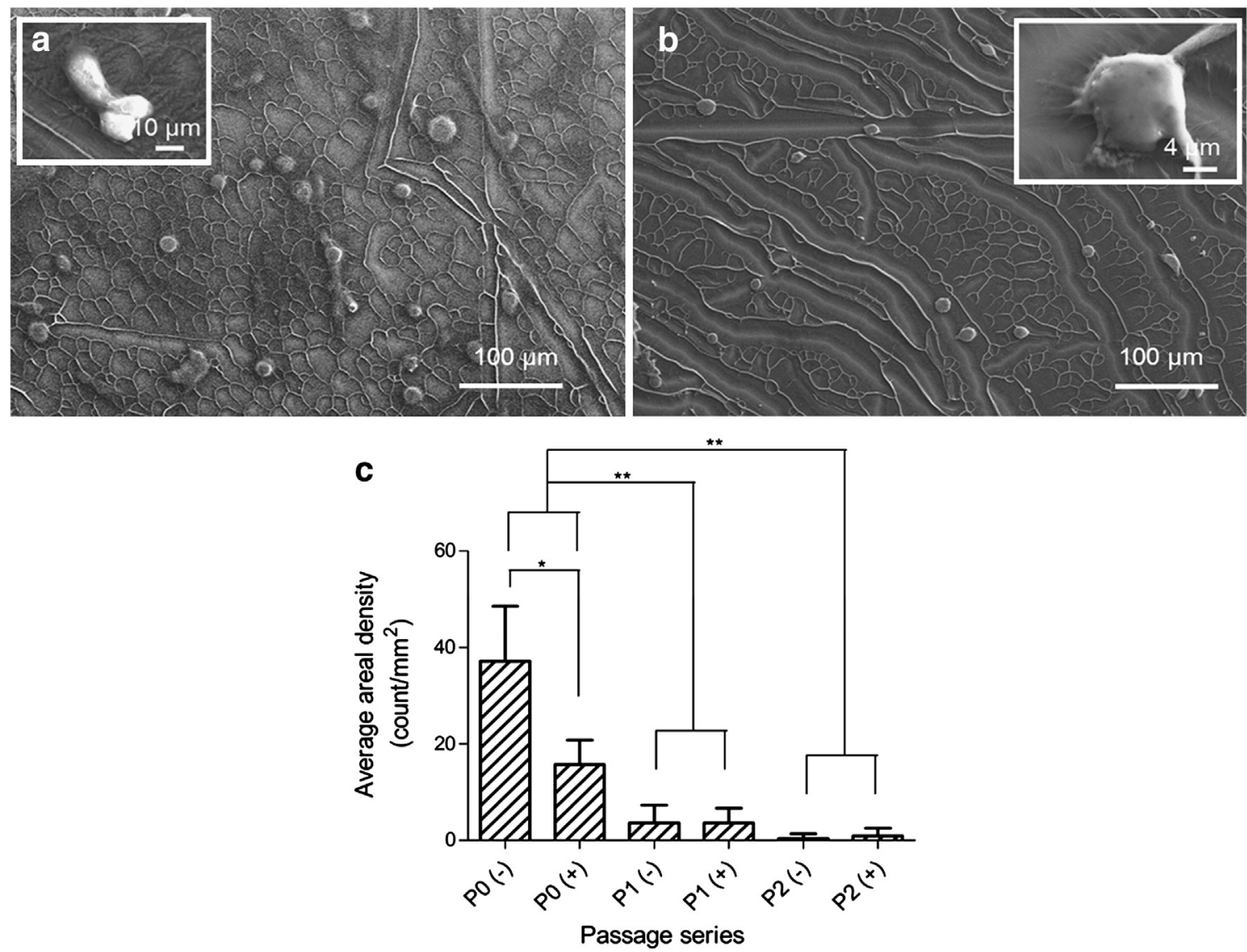

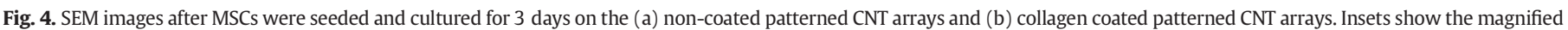

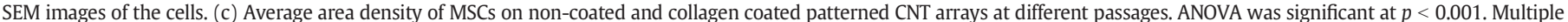
comparisons of control to other groups were performed using Fisher's test (Minitab 15 Statistical Software). ${ }^{*}$ indicates significant $p<0.05$.

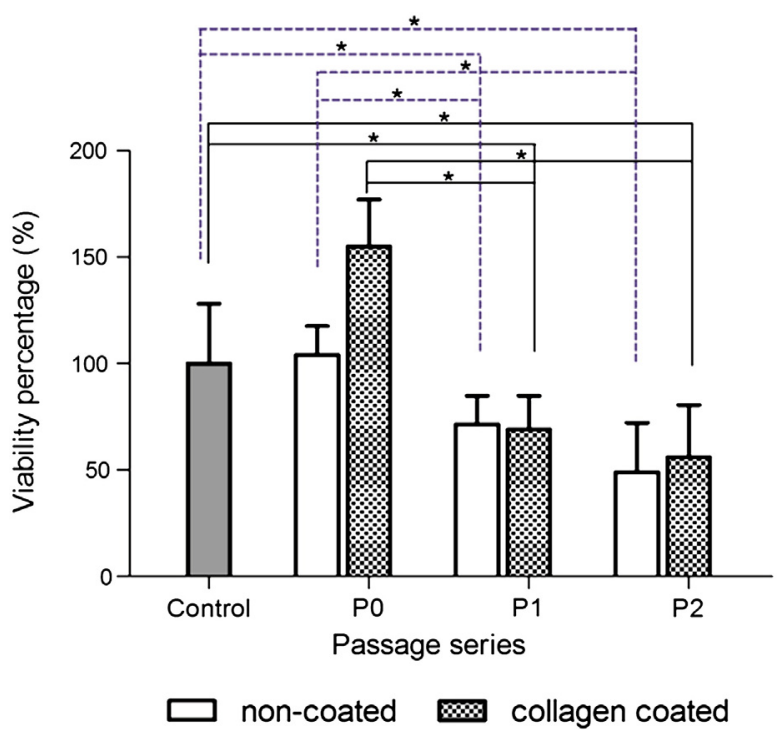

Fig. 5. MTT assay graphs showing the percent viability of MSCs on the non-coated patterned CNT arrays (empty bars) and collagen coated patterned CNT arrays (solid bars) at different passages. Dotted blue lines indicate the comparison of viability percentage on the non-coated CNT surfaces. Solid lines show the comparison of viability percentage on the collagen coated CNT surfaces. ANOVA was significant at $p=0.041$ and $p<0.001$ for non-coated and collagen coated CNT arrays, respectively. Multiple comparisons of control to other groups were performed using Fisher's test (Minitab 15 Statistical Software). * indicates significant $p<0.05$ would be to embed CNTs while preserving their alignment in a biodegradable matrix manufactured from chitosan, polylactic acid, etc. [40]. In this concern, our studies regarding the transfer of aligned CNT arrays to a polymer matrix are ongoing.

\section{Conclusion}

We investigated MSC viability on bare and collagen-coated patterned CNT arrays. First, our results revealed that MSCs attached to both non-coated and collagen coated patterned CNT arrays better than on bare 96-well culture plates for the early passage (P0). Moreover, we did not encounter evidence for the toxicity of CNTs. However, cell numbers and viability decreased significantly on both coated and non-coated patterned CNT surfaces for later passages (P1 and P2). Furthermore, we found that collagen coating of CNTs had an impact on the adhesion of MSCs. This may suggest that MSC adhesion could be influenced by topography of the surface. Overall, this study demonstrated that patterned CNT arrays can serve as a permissive scaffold for MSC growth and adhesion. CNTs are not biodegradable and as such that they can be used as scaffolds where long-term substrates are needed for tissue engineering such as in regeneration after spinal cord or brain injury.

\section{Acknowledgements}

This work was supported partly by the Scientific and Technological Research Council of Turkey (TUBITAK) grants SBAG105S393 and 
SBAG106S460 to K.C. Akcali, V. Bitirim and G. Kucukayan-Dogu thank TUBITAK for financial support. The authors are grateful to Rasit Turan and Mustafa Kulakci for electron beam evaporation facility at Middle East Technical University (Turkey).

\section{References}

[1] Z. Tokcaer-Keskin, A.R. Akar, F. Ayaloglu-Butun, E. Terzioglu-Kara, S. Durdu, U. Ozyurda, M. Ugur, K.C. Akcali, Can. J. Physiol. Pharmacol. 87 (2009) 143-150.

[2] A.J. Nauta, W.E. Fibbe, Blood 15 (2007) 3499-3506.

[3] P.X. Ma, Adv. Drug Deliv. Rev. 60 (2008) 184-198.

[4] W. Li, R. Tuli, X. Huang, P. Laquerriere, R.S. Tuan, Biomaterials 26 (2005) 5158-5166.

[5] J.L. Ifkovits, J.J. Devlin, G. Eng, T.P. Martens, G. Vunjak-Novakovic, J.A. Burdick, Appl. Mater. Interfaces 1 (2009) 1878-1886.

[6] M.J. Dalby, N. Gadegaard, R. Tare, A. Andar, M.O. Riehle, P. Herzyk, C.D.W. Wilkinson, R.O.C. Oreffo, Nat. Mater. 6 (2007) 997-1003.

[7] D.R. Jung, R. Kapur, T. Adams, K.A. Giuliano, M. Mrksich, H.G. Craighead, D.L. Taylor, Crit. Rev. Biotechnol. 21 (2001) 111-154.

[8] F. Gelain, S. Panseri, S. Antonini, C. Cunha, M. Donega, J. Lowery, F. Taraballi, G. Cerri, M. Montagna, F. Baldissera, A. Vescovi, ACS Nano 5 (2011) 227-236.

[9] T.D.B. Nguyen-Vu, H. Chen, A.M. Cassell, R.J. Andrews, M. Meyyappan, J. Li, IEEE Trans. Biomed. Eng. 54 (2007) 1121-1128.

[10] M.J. Dalby, S.J. Yarwood, M.O. Riehle, H.J. Johnstone, S. Affrossman, A.S. Curtis, Exp. Cell Res. 276 (2002) 1-9.

[11] T.P. Kunzler, C. Huwiler, T. Drobek, J. Voros, N.D. Spencer, Biomaterials 28 (2007) 5000-5006.

[12] F. Gentile, L. Tirinato, E. Battista, F. Causa, C. Liberale, E.M. di Fabrizio, P. Decuzzi, Biomaterials 31 (2010) 7205-7212.

[13] E. Hirata, M. Uo, H. Takita, T. Akasaka, F. Watari, A. Yokoyama, Carbon 49 (2011) 3284-3291.

[14] R.J. Chen, S. Bangsaruntip, K.A. Drouvalakis, N.W.S. Kam, M. Shim, Y. Li, W. Kim, P.J. Utz, H.J. Dai, PNAS 100 (2003) 4984-4989.

[15] C.A. Poland, R. Duffin, I. Kinloch, A. Maynard, W.A. Wallace, A. Seaton, V. Stone, S. Brown, W. MacNee, K. Donaldson, Nat. Nanotechnol. 3 (2008) 423-428.

[16] K. Soto, K.M. Garza, L.E. Murr, Acta Biomater. 3 (2007) 351-358.

[17] G. Jia, H. Wang, L. Yan, X. Wang, R. Pei, T. Yan, Y.L. Zhao, X.B. Guo, Environ. Sci. Technol. 39 (2005) 1378-1383.
[18] S.K. Smart, A.I. Cassady, G.Q. Lu, D.J. Martin, Carbon 44 (2006) 1034-1047.

[19] L. Zhu, D.W. Chang, L. Dai, Y. Hong, Nano Lett. 7 (2007) 3592-3597.

[20] A.O. Lobo, M.A.F. Corat, E.F. Antunes, M.B.S. Palma, C. Pacheco-Soares, E.E. Garcia E.J. Corat, Carbon 48 (2010) 245-254.

[21] S. Giannona, I. Firkowska, J. Rojas-Chapana, M. Giersig, J. Nanosci. Nanotechnol. 7 (2007) 1679-1683.

[22] P.R. Supronowicz, P.M. Ajayan, K.R. Ullmann, B.P. Arulanandam, D.W. Metzger, R. Bizios, J. Biomed. Mater. Res. 59 (2002) 499-506.

[23] X. Zhang, S. Prasad, S. Niyogi, A. Morgan, M. Ozkan, C.S. Ozkan, Sensors Actuators B 106 (2005) 843-850.

[24] E. Money, P. Dockery, U. Greiser, M. Murphy, V. Baron, Nano Lett. 8 (2008) 2137-2143.

[25] M.M. Bradford, Anal. Biochem. 72 (1976) 248-254.

[26] C.T. Wirth, S. Hofmann, J. Robertson, Diam. Relat. Mater. 17 (2008) 1518-1524.

[27] Z. Tokcaer-Keskin, Z.G. Dikmen, F. Ayaloglu-Butun, S. Gultekin, S.M. Gryaznov, K.C. Akcali, Stem Cell Rev. 6 (2010) 224-233.

[28] A.A. Mangi, N. Noiseux, D. Kong, H. He, M. Rezvani, J.S. Ingwall, V.J. Dzau, Nat Med. 9 (2003) 1195-1201.

[29] Y. Cho, R.B. Borgens, J. Biomed. Mater. Res. A 95A (2010) 510-517.

[30] E. Hirata, M. Uo, H. Takita, T. Akasaka, F. Watari, A. Yokoyama, J. Biomed. Mater. Res. B 90B (2009) 629-634.

[31] R.A. MacDonald, B.F. Laurenzi, G. Viswanathan, P.M. Ajayan, J.P. Stegemann, J. Biomed. Mater. Res. A 74 A (2005) 489-496.

[32] I. Sridharan, T. Kim, R. Wang, Biochem. Biophys. Res. Commun. 381 (2009) 508-512.

[33] A.J. Engler, S. Sen, H.L. Sweeney, D.E. Discher, Cell 126 (2006) 677-689.

[34] E.E. Da Silva, H.H.M.D. Colleta, A.S. Ferlauto, R.L. Moreira, R.R. Resende, S. Oliveira, G.T. Kitten, R.G. Lacerda, L.O. Ladeira, Nano Res. 2 (2009) 462-473.

[35] C. Li, T.-W. Chou, Compos. Sci. Technol. 63 (2003) 1517-1524.

[36] D. Liu, C. Yi, D. Zhang, J. Zhang, M. Yang, ACS Nano 4 (2010) 2185-2195.

[37] B.R. Twaites, C.H. Alarcon, M. Lavigne, A. Saulnier, S. Pennadam, D. Cunliffe, D.C Gorecki, C.J. Alexander, J. Control. Release 108 (2005) 472-483.

[38] H. Gruber, S. Somayaji, F. Riley, G. Hoelscher, H. Norton, J. Ingram, E.N. Hanley, Biotech. Histochem. 87 (2012) 303-311.

[39] P.R. Crisostomo, M. Wang, G.M. Wairiuko, E.D. Morrell, A.M. Terrell, P. Seshadri, U.H. Nam, D.R. Meldrum, Shock 26 (2006) 575-580.

[40] G. Bayramoglu, K.C. Akcali, S. Gultekin, E. Bengu, M.Y. Arica, Macromol. Res. 19 (2011) 385-395. 